\title{
Zur Morphologie des Glykogens des Herzmuskels nebst Bemerkungen über dessen Struktur.
}

\author{
Von
}

Professor Dr. Julius Arnold in Heidelberg.

Hierzu Tafel XXVIII.

\section{Die Morphologio des Glykogens.}

Die Untersuchung der Skelett- und Bauchmuskeln (Nr. 3) hat. ergeben, dass das Glykogen hauptsächlich, wenn nicht ausschliesslich, in den Sarkosomen enthalten ist, während solches in den Muskelfibrillen, wenigstens in den anisotropen Abschnitten Q den sogenannten Myokonten - nicht nachgewiesen werden kann. Die an der Stelle der isotropen Scheiben - J - gelegenen. transversalen Glykogengranula wurden als sarkoplastische Gebilde aufgefasst; dafür sprechen ihre Beziehung zu dem intercolumnären glykogenführenden Sarkoplasma, mit welchem sie $Q$ umspinnende Netze bilden, sowie ihr vermutlich vom Kontraktionszustand abhängiger Lagewechsel zwischen $Q$ und der Zwischenscheibe $Z$; in Folge dieses erscheinen die transversalen Granula als eine einfache $\mathrm{Z}$ verdeckende Reihe oder aber in der Form von zwei Reihen, welche in verschiedener Entfernung von $Z$ liegen, manchmal aber auch $Q$ sich nähern dieses mehr oder weniger verdeckend.

Bei der supravitalen Jodräucherung (Brusthautmuskel) zeigen zunächst nur die Sarkosomen die Glykogenreaktion; diffuseFärbungen im Sarkoplasma treten gewöhnlich erst später auf; die Muskelfibrillen bleiben ungefärbt und nehmen nur einen bellgelben Farbenton an.

Wie bei den Skelettmuskeln, so stimmen auch bei dem Herzmuskel die Angaben über das Verhalten des Glykogens nicht überein; die Einen verlegen den Sitz dieses in das Sarkoplasma, die anderen in das Myoplasma, die meisten schreiben ihm eine diffuse Verteilung zu und beziehen das Vorkommen von Körnchen, mögen sie diese in das erstere oder letztere verlegen, auf Fallungsvorgånge. Es war somit eine Untersuchung des Herz- 
muskels nicht zu umgehen und eine Prüfung in der Hinsicht erforderlich, ob und in wie fern die Anordnung des Glykogens im Herzmuskel von derjenigen in den Skelettmuskein abweicht.

Ich habe mich vorerst auf die Untersuchung des Froschherzens beschränkt, da es mir sachgemäss erschien, zunächst bei der gleichen Art die Anordnung des Glykogens in den Skelettmuskeln einerseits, den Muskelfasern des Herzens andererseits festzustellen. Ich hoffe seinerzeit über die Morphologie des Glykogens im Herzen der Warmblüter unter besonderer Berücksichtigung der Purkinjeschen Fasern berichten zu können Uebrigens darf das Froschherz nicht nur wegen seines gewöhnlich ziemlich beträchtlichen Glykogengehaltes als Untersuchungsobjekt empfohlen werden; ein weiterer Vorzug ist der, dass man dieses in toto $\mathrm{d}$. $\mathrm{h}$. ohne Zerlegung in kleinere Stücke in absolutem Alkohol härten und in Zelloidin einbetten kann.

Die Methoden waren im wesentlichen die gleichen, wie bei den früheren Glykogenuntersuchungen. Zunächst prüfte ich das Verhalten des frischen Herzmuskels bei der Jodriucherung. Möglichst kleine Stückchen wurden in der Glaskammer (hohlgeschliffenem Objektenträger) Joddåmpfen ausgesetzt. Die Ergebnisse waren aber weniger befriedigend wie bei den Skelettmuskeln; es mag dies seinen Grund darin haben, dass man genötigt ist, Zupf- oder Schnittpräparate anzufertigen; begreiflicher Weise ist der vorsichtig von seinen Ansatzstellen abgelöste und in seiner Form gut erhaltene Brusthautmuskel ein brauchbareres Versuchsobjekt. Immerhin findet man auch im Herzmuskel eine bald grössere bald kleinere Zahl von Fasern mit jodophilen Sarkosomen, während die eigentlichen Fibrillen ungefärbt bleiben. Es sei noch bemerkt, dass man auch an Schnitten von Präparaten, welche in Zelloidin eingebettet und nach vorausgehender Entwässerung mit absolutem Alkohol durch Origanumöl aufgehellt wurden, mittelst der Jodräucherung sehr brauchbare Bilder erhält. Diese Methode angewendet auf das frische Objekt, ist insofern, wie schon mehrfach hervorgehoben wurde, von Bedeutung, als mit ihrer Hilfe nachgewiesen werden kann, dass vorwiegend die Sarkosomen das Glykogen enthalten; diffuse Färbungen des Sarkoplasma habe ich erst später wahrgenommen, wăhrend die eigentlichen Muskelfibrillen auch in dieser Zeit ungefărbt bleiben. Überhaupt sollte die Anfertigung von Jod- 
präparaten niemals unterlassen werden; nur darf man nicht vergessen, dass bei Anwendung von wässerigen Jodlösungen selbst am gehärteten Objekt noch Veränderungen des Glykogens eintreten können. Solche kommen auch an Präparaten vor, welche nach der Bestschen Karminmethode, die ja sonst viel leistungsfảhiger ist, behandelt wurden. - Manchmal ist, wie Querschnitte durch das Herz lehren, die Verteilung des Glykogens eine ungleichmăssige in der Art, dass auf der einen Seite sehr wenig, auf der anderen Seite sehr viel Glykogen in dem Herzmuskel sich findet. Hängt man das Herz in absolutem Alkohol auf, so macht sich diese Unregelmăssigkeit, welche offenbar auf durcb die Konservierung bedingte Diffusionsströme zurückzuführen ist, viel weniger bemerkbar. Der Befund von kleineren und grösseren freien, d. h. nicht an Strukturbestandteile gebundenen Tropfen ist wohl gleichfalls auf Lösungserscheinungen $\mathbf{z u}$ beziehen; freie körnige Abscheidungen und Fällungen habe ich bei richtigem Verfahren nicht beobachtet. Bei allen diesen Vorkommnissen spielen die wechselnden Lösungsverhältnisse des Glykogens, die vitalen und postvitalen autolytischen Vorgänge, die angewandten Untersuchungsmethoden eine hervorragende Rolle. Um so unentbehrlicher ist, wie ich in den früheren Mitteilungen immer wieder betont habe, die Controle am überlebenden Objekte. Wie oben erwähnt wurde, kommen nach einiger Zeit auch an diesem diffuse Färbungen zur Wahrnehmung; in wie weit diese als vitale, postvitale oder arteficille Erscheinungen aufzufassen sind, lasst sich am konservierten Präparate nicht feststellen.

Wie in den Skelettmuskeln, so kann man auch an den Herzmuskeln ein longitudinales und ein transversales System vor glykogenfübrenden Granula unterscheiden.

Das erstere entspricht den intercolumnären Räumen und zeigt einen durch den verschiedenen Glykogengehalt bedingten Wechsel in der Anordnung der glykogenführenden Sarkosomen (Fig. 1-3). Bald sind diese nur spärlich und klein, in kurzen und vielfach unterbrochenen Reihen aufgestellt, bald sind sie zahlreicher, grösser, bilden lange und zuweilen breite Reihen, sowie auf grössere Strecken hin zusammenhängende Ketten. Manchmal sind einzelne Sarkosomen und deren Zwischenglieder überhaupt nicht mehr zu unterscheiden. Dazwischen finden sich kleinere und grössere freie Tropfen. Die Verbindung der Sar- 
kosomen, wenn eine solche nachweisbar ist, wird bald durch gefürbte bald durch ungefurbte fädige $\mathrm{Zwischenglieder}$ hergestellt. Wesentliche Unterschiede gegenüber der Anordnung in den Skelettmuskeln wüsste ich nicht hervorzuheben. Die Gliederung der intercolumnären Glykogensubstanzen in Sarkosomenreihen erschien mir am Herzmuskel eher deutlicher als an den Skelettmuskeln. Auf dem Durchschnitt habe ich eine netzförmige Anordnung des glykogenbaltigen Sarkoplasmas nur stellenweise, dagegen zahlreiche dicht stehende Granula zwischen den querdurchschnittenen Fibrillenkomplexen beobachtet.

Die Kerne sind stets frei von Glykogen, dagegen finden sich solche Granula in dem umgebenden Sarkoplasma in grösserer Zahl (Fig. 1).

Was das transversale System der Glykogengranula anbelangt, so zeigt auch dieses wechselnde Anordnung: bei geringerem Glykogengehalt vereinzelte Granula, deren Lage zu den einzelnen Fibrillen und Fibrillenabschnitten schon wegen der geringeren Breite dieser, sowie wegen der Verwechslung mit intercolumnaren Sarkosomen nicht immer mit Sicherheit $z u$ bestimmen ist. Leichter wird dies bei grösserem Glykogengehait. Am hãufigsten nimmt man an der Stelle der Zwischenscheibe (Z) eine bald sebr schmale, bald etwas breitere rote Linie wahr, welche eine Zusammensetzung aus Granula namentlich dann erkennen lässt, wenn die letzteren weniger dicht liegen; sehr oft erscheint sie mehr gleichartig (Fig. 3). Man erhalt so den Eindruck, als ob $Z$ aus Glycogengranula bestehe. An den Skelettmuskeln konnte nachgewiesen werden, dass an der Stelle der isotropen Substanz $(J)$ zu beiden Seiten von $Z$ Reihen von Glykogengranula sich finden, welche zuweilen dem letzteren näher rücken und es schliesslich verdecken. Während in dem letzteren Falle der Anschein erweckt werden kann, als ob Z Glykogengranula enthielte, lässt sich nachweisen, dass $Z$, wenn die Glykogengranula an der Stelle von J gelegen sind, eine Glykogenreaktion nicht darbietet. Ich kam deshalb zu dem Schluss, dass $\mathrm{Z}$ glykogenfrei ist und ein Glykogengehalt dieses durch die Verlagerung der J-granula vorgetauscht wird.

Bei eingehender Prüfung konnte ich auch am Herzmuskel Fasern auffisden, bei welchen $\mathrm{Z}$ ungefärbt zwischen den zwei Reihen der Glykogengranula $J$ nachgewiesen werden konnte 
(Fig. 3 b). Der Wechsel in der Lagerung dieser hangt offenbar mit dem verschiedenen Kontraktionszustand zusammen. - Wie früher hervorgehoben wurde, kommt es bei den Skelettmuskeln sehr baufig durch eine Vereinigung der longitudinalen und transversalen Granulasysteme zur Bildung mehr oder weniger regelmässiger Netze, welche die Felder $Q$ diese gewöhnlich freilassend umsäumen. An den Herzmuskeln finden sich solche Netze gleichfalls (Fig. 2c), sie scheinen aber seltener zu sein; doch kommt dieser Verschiedenheit liaum eine Bedeutung zu, weil sie vermutlich nur gradueller Art ist, d. h. von dem Glykogengehalt abhängt. Es wurde eben betont, dass an der Stelle der Felder Q Glykogen meistens vermisst wird; wenn das Sarkoplasma von den Seiten her über $Q$ sich wegschiebt, so kann ein Glykogengehalt dieser vorgetäuscht werden.

Ob die Endothelien Glykogen führen, kann ich nicht mit Bestimmtheit aussagen, weil im Blut sehr oft freie Glykogentropfen sich finden, welche dem Endothel möglicherweise sich autlagern. Dagegen liess sich in den Leukocyten, insbesondere auch in den eosinophilen Formen Glykogen nachweisen; doch waren diese weniger zahlreich wie im Blut der Lebergefiusse.

Es wurde erwähnt, dass der Sitz des Glykogens von den Einen in das Myoplasma, von den Anderen in das Sarkoplasma verlegt wird, die Meisten aber annehmen, dass seine Verteilung eine diffuse und der Befund von Körnchen auf Fallungsvorgänge zurückzuführen. sei. - Die oben mitgeteilten Untersuchungen haben uns zu dem Ergebnis geführt, dass in den Muskelfibrillen Glykogen überhaupt nicht enthalten ist; die transversalen an der Stelle der isotropen Substanz gelegenen Granula gebören dem Sarkoplasma an; allerdings kann durch die Verlagerung dieses das Trugbild gefärbten Myoplasmas entstehen. - Die im Sarkoplasma gelegenen Körner als Fällungsprodukte aufzufassen, dagegen sprechen ausser den früher geschilderten Befunden bei der supravitalen Jodrăucherung die regelmässige Anordnung der longitudinalen und transversalen Systeme der Glykogengranula, insbesondere aber, wie weiter unten nachgewiesen werden soll, ihre vollstandige Übereinstimmung mit der Anordnung der Sarkosomen, wie sie am überlebenden und nach verschiedenen Methoden conservierten Objekt beobachtet werden kann. Diffuse Färbungen des Sarkoplasmas kommen vor; es wurde oben erörtert, weshalb 
die Entscheidung, ob es sich um eine vitale, postvitale, oder arteficielle Erscheinung handelt, zur Zeit nicht möglich ist. Wer an gelungenen Glykogenpräparaten mit den zierlichen und bei allem Wechsel gesetzmässigen Anordnungen der longitudinalen und transversalen Granulasysteme sich vertraut gemacht hat, wird zu der Überzeugung gelangen, dass das Glykogen an präexistente Strukturbestandteile gebunden ist.

\section{Zur Struktur des Herzmuskels.}

Die Methoden der Untersuchung waren im wesentlichen die gleichen, wie bei derjenigen der Skelettmuskeln: Konservierung in Alkohol, Sublimatchlornatriumlösungen oder Bend a scher Chromosmiummischung (Nachbehandlung mit Acetum pyrolignosum und Chromsäure etc.). Von Tinktionsmetboden kamen in Anwendung ausser den gewöhnlicben die Eisenhämatoxylinfärbung ohne und mit nachträglicher Tinktion durch Krystallviolettanilinöl; in letzterem Fall müssen die durch Eisenhämatoxylin gefärbten Objekte stark differenziert werden. Sehr brauchbar erwies sich folgende Modifikation der Bendaschen Mitochondrienfirbung: nach volizogener Tinktion mit Krystallviolettanilinöl trocknet man die Präparate ab und differenziert ohne Anwendung von Essigsäure mit einer Mischung von Aceton-Nelkenöl $(2: 10)$, bis die Felderzeichnung an den Muskelfasern deutlich wird. Dünne Paraffinschnitte $(2-4, \mu)$ sind unbedingt erforderlich. Ich will nicht unterlassen, auch an dieser. Stelle zu betonen, dass es sich bei diesen Färbungen nicht um "spezifische" handelt; vielmehr ist ihr Ergebnis von dem Grad der Differenzierung und dem Kontraktionszustand abhängig.

Sarkoplasma. Zunächst sei erwähnt, dass bei vitaler Zufuhr von Methylenblau in der Muskulatur des noch lebbaft sich kontrahierenden Froschherzens gefarbte Granula vorkommen. Dieselben liegen zwischen den Fibrillensystemen. Ich verweise auf meine früheren Mitteilungen (Nr. 2). Mittelst der Jodkalimaceration lassen sich die Muskelsăulchen, später die Muskelfibrillen isolieren. Die Sarkosomen kommen dann sehr deutlich zum Vorschein (Nr. 1).

Nach den an den Glykogenprăparaten erhobenen Befunden war zu erwarten, dass in der Muskulatur des Froschherzens das Sarkoplasma ziemlich reichlich vertreten, aber in seiner 
Anordnung einem ziemlich grossen Wechsel unterworfen ist. Es fanden sich an den nach den eben erwalunten Methoden behandelten Objekten bald nur vereinzelte kleinere und grössere Granula, bald Ketten und Reihen solcher, welche in den interkolumnaren Ralumen. lagen (Fig. 4-6). Sehr oft waren sie durch feine Faden verbunden oder es bot sich mehr das Bild von Fäden dar, welche in regelmãssigen Abständen durch Granula von wechselnder Grösse und Farbung unterbrochen wăren. Die grösseren Granula zeigten einen mehr dunkelbraunen bis schwärzlichen, die kleineren einen helleren Farbenton mit einer mehr oder weniger deutlichen Beimischung won blau, wenn Krystallviolett in Anwendung gekommen war. Die einen Granula schienen zwischen Q, ohne zu diesen in Beziehung zu treten, die anderen in der Höhe von $Z$ zu liegen, und mit diesen durch fädige Fortsatze in Verbindung zu stehen (Fig. j). Da, wie nachher berichtet werden soll, auch die an der Stelle von $Z$ gelegenen Gebilde nach diesen Methoden sich fürben, so entstehen netzförmige, die Muskelsegmente (Muskelkastchen) umsäumende Figuren (Fig. 5 b). Unregelmässigkeiten in der Anordnung dieser sind wohl auf artefizielle Einwirkungen zurückzufülrren (Fig. jc). - Es ist wohl nicht erforderlich, auf eine Vergleichung dieser Bilder mit den an Glykogenpriparaten beobachteten einzugehen und ihre C̈bereinstimmung ausführlicher zu erörtern; dagegren sei noch hervorgehoben, dass eine solche auch bezüglich der Darstellungen, welche andere von dem Verhalten des Sarkoplasmas im Herzmuskel geben, verzeichnet werden darf. Ich verweise insbesondere auf die diesen Gegenstand betreffenden Schilderungen Heidenhains.

Myofibrillen, Myokonten und Myosomen. Wederan Alkohol-, noch an Sublimat- oder Chromosmiumpräparaten erhalt man den Eindruck von begrenzten Zellen, vielmebr den von Faserbündeln, welche aber keine Abgrenzung erkennen lassen. Besonders geeignet zum Studium der Myofibrillell sind nach den oben angegebenen Methoden gefărbte Chromosmiumobjekte. Je nach dem Grad der Differenzierung, insbesondere aber je nach dem Kontraktionszustand der Fasern ergeben sich sehr wechselnde Bilder. Sehr auffallend war mir, dass man zuweilen in dem gleichen Faserbündel verschiedene Kontraktionszustände der Fasern wahrnehmen kann. 
Manche Fasern sind gleichmässig gefürbt und es ist eine Gliederung an ihnen nicht zu beobachten Andere zeigen eine regelmässige Felderung; es entstehen, durch $Z$ begrenzt, Segmente, welehe aus dem gefärbten $Q$ und den beiden ungefärbten $J$ sich zusammensetzen (Fig. 7 a u. b). An Präparaten, welche nach der modifizierten Bendaschen Methode tingiert wurden, erschienen die Felder $\mathrm{Q}$ dunkelviolett, $\mathrm{Z}$ blau, bei der Färbung mit Eisenhämatoxylin und Krystallviolettanilinöl die ersteren schwarzblau, $Z$ graublau. Bei sehr vielen Fasern macht sich offenbar infolge der Lockerung der Interfibrillarsubstanz an diesen Segmenten die Neigung, in feinere Stäbchen zu zerfallen, bemerkbar; an diesen kann man den mittleren, etwas dickeren gefurbten, Abschnitt, welcher $Q$ entspricht, und seine ungefärbten Fortsätze unterscheiden (Fig. 8). So entstehen primitive, aus J-Q-J bestehende Fibrillensegmente. Ob die Fibrillen an der Stelle von $Z$ diese kontinuierlich durchsetzen oder an dieser Stelle durch Granula unterbrochen werden, konnte ich nicht mit Sicherheit feststellen. An isolierten Primitivfibrillen findet sich entsprecbend $Z$ ein granulaartiges Gebilde (Fig. 8 b u.c). Wie an den Skelettmuskeln lassen sich auch an Primitivfibrillen des Herzens metamer angeordnete Fibrillensegmente nachweisen. Während durch ihre Anordnung in der Langsrichtung Primitivfibrillen entstehen, werden durch ibre quere Verbindung Muskelsegmente oder Muskelkästchen, durch ibr Aneinanderreihen in der Längsrichtung Muskelsäulchen gebildet (Fig. 8). Ob diese, wie $\mathrm{Schlater}$ in seiner bemerkenswerten Arbeit hervorhebt, immer nur aus zwei Primitivfibrillen bestehen, wage ich nicht zu entscheiden. Kommt es $\mathrm{zu}$ einer vollständigen Isolierung der Primitivfibrillen, so ist man durch die Feinheit dieser überrascht. Bei den stärksten Vergrösserungen stellen sie sich als dünnste Fäden, welche in ihrem Verlauf oft nur durch den gefärbten Abschnitt Q - den Myokont - und das granulaartige Gebilde an der Stelle von $\mathrm{Z}$ zu verfolgen sind (Fig. 8d), dar. Bei stárkerer Differenzierung kommen an der Stelle des Myokonten Granula zum Vorschein. Bei den Skelettmuskeln gelang mir der Nachweis, dass in den beiden Enden des Myokonten je ein distinktes Granulum enthalten ist, nachdem schon Schlater an der Stelle von $\mathrm{Q}$ das Vorkommen eines Doppelgranulums eingehend beschrieben hatte. Die Existenz eines solchen nimmt er im. 
Gegensatz zu Heidenhain in den Muskelfibrillen des Herzens (Hühnerembryo) an. Ich muss bekennen, dass ich wegen der Kleinheit des Objekts und der Feinheit der Fibrillen beim Froschherzen darüber mir nicht Gewissheit verschaffen konnte, ob die Myokonten ein oder zwei Granula-Myosomen enthalten. Die Entscheidung dieser Frage wird auch dadurch erschwert, dass bei stärkerer Differenzierung $Z$ sich zu entfärben beginnt und es sehr schwierig wird, die Lage der Granula in den Fibrillensegmenten zu bestimmen. In einzelnen Fibrillen glaube ich an der Stelle der Myokonten zwei Granula wahrgenommen zu haben.

Ganz andere Bilder bieten andere Fasern dar. Zwischen bald feineren bald dickeren intensiv gefärbten Scheiben, welche an der Stelle von $Z$ zu liegen scheinen, finden sich hellere schwächer tingierte Abschnitte von wechselnder Dicke. Sie lassen eine Zusammensetzung aus J-Q-J nicht erkennen (Fig. 9).

Dass diese verschiedene Erscheinung der Muskelfibrillen der Ausdruck verschiedener Kontraktionszustïnde und eines der interessanten Beispiele von funktionellem Strukturwechsel ist, liegt auf der Hand. Den Versuch der Deutung und Bewertung in der letzteren Hinsicht möchte ich elst unternehmen, wenn meine Erfahrungen durch die Untersuchung des Warmblüterherzens bereichert sind. Auch die Literatur soll dann eingehender berïcksichtigt werden.

\section{Ergebnisse.}

1. Wie in den Skelettmuskeln ist auch in den Muskelfasern des Froschherzens das Glykogen an die Sarkosomen gebunden.

2. Die Muskelfibrillen des Herzens enthalten kein Glykogen.

3. Die Primitivfibrillen bestehen aus Segmenten, welche sich aus J-Q-J zusammensetzen und durch $Z$ begrenzt werden.

4. Durch Aneinanderreihung solcher Fibrillensegmente in der Längsrichtung entstehen Primitivfibrillen, in der Querrichtung Muskelkästchen oder Muskelsegmente.

5. Die gefärbten Abschnitte $Q$ der Fibrillensegmente die Myokonten - enthalten Granula - Myosomen -; ob je zwei oder nur eines ist fraglich. 


\section{Literaturverzeichnis.}

1. Arnold, J.: Über feinere Struktur und Architektur der Zellen. Teil III Muskelgewebe. Arch. f. mikr. Anat., Bd. 52, 1898.

2. Derselbe: Über vitale Granulafärbung in den Knorpelzellen, Muskelfasern etc. Arch. f. mikr. Anat., Bd. 55, 1901.

3. Derselbe: Zur Morphologie des Muskelglykogens etc. Arch. f. milkr. Anat., Bd. 73, 1909.

4. Aschoff: Über den Glykogengehalt des Reizleitungssystems des Säugetierherzens. Verhandl. d. patholog. Gesellschaft, 1908.

5. Dietrich: Über die Querlinien der Herzmuskeln. Zentralbl. f. allgem. Patholog., 1906 u. Verhandl. d. patholog. Gesellsch., 1906.

6. Ebner: Über die Kittlinien der Herzmuskelfasern. Sitzungsber. d. Akadem. d. Wissensch. in Wien, math.-naturw. Kl., Abt. III, Bd. 109, 1900.

7. Fusari: Sur la structure des fibres musculaires striées. Arch. ital. d. Biolog., Bd. 23, 1894.

8. Gàdzikiewicz: Über den feineren Bau des Herzens etc. Jenaer naturw. Zeitschr., Bd. 39, 1904.

9. Godlewsky: Über die Entwicklung des quergestreiften Muskelgewebes. Arch. f. mikr. Anat, Bd. 60, 1902.

10. Heidenhain, M.: Beiträge zur Aufklärung des wahren Wesens der faserförmigen Differenzierungen. Anat. Anz., Bd. 16, 1899.

11. Derselbe: Struktur der kontraktilen Materie, Ergebn. f. Anat. u. Entwicklungsgesch, Bd. VIII, $1898_{i} 99$ u. Bd. X, 1901.

12. Derselbe: Über die Struktur des menschlichen Herzmuskels. Anat. Anz., Bd. 20, 1902.

13. Heubner: Die Spiralwindung der Herzmuskelkerne. Deutsch. Arch. f. lilin. Medizin, Bd. 88, 1907.

14. Hoche: Recherch. sur la structure des fibr. muscul. cardiaq. Bibliograph. Anatomie, 1891.

15. Holmgren, E.: Über die Sarkoplasmakörner quergestreifter Muskel-fasern. Anat. Anz., Bd. 31, 1907.

16. Hoyer: Über die Kontinuität der kontraktilen Fibrillen in den Herzmuskelzellen. Anz. d. Akad. d. Wissensch. Krakau, math.-naturw. Kl., Nr. 3, 1901.

17. Mac-Callam, J. B.: On the histolog. and histogen. of the heart muscle cell. Anat. Anz., Bd. 13, 1897.

18. Mader. Sur les fibres musculaires du coeur etc. C. R. Acad. Scienc. Paris, T. $138,1904$.

19. Marcean: Recherch. sur l'histolog. et le developpement comparées des fibres Purkinje etc. C. R. soc. biolog., T. 53, 1902.

20. Derselbe: Note sur la structure des fibres muscul. cardiaq. chez les oiseaux. C. R. soc. biol., T. 54, 1902.

21. Derselbe: Recherch. sur les bandes transversales scalariformes etc. C. R. Acad. Scienc., T. 136, 1903. 
22. Derselbe: Recherch. sur la constitution et sur la structure des fibres cardiaq. chez les vertébrés inferieurs. C. R. Acad. Scienc. Paris, T. 136, 1903.

23. Derselbe: Recherch. sur la structure et le developpement comparées des fibres cardiaq. dans la serie des vertébrés. Annal. scienc. natur. Ser. 8 , Zoolog., T. 19, 1903.

24. Derselbe: Note sur la structure du coeur chez les Cephalopodes. Bull. soc. d'hist. natur. Doubs, 1904.

25. Derselbe: Recherch. sur la structure du coeur chez les mollusques etc. Arch. d'anat. microscop., T. 7, 1906.

26. Marchand: Über eine Geschwulst aus quergestreiften Muskelfasern mit ungewöhnlichem Gehalt an Glykogen etc. Virch. Arch., Bd. 100, 1885.

27. Moenckeberg: Über die sgt. abnormen Sehnenfäden etc. Verh. d. pathol. Gesellsch., 1908.

28. Moriga: Über die Muskulatur des Herzens. Anat. Anz., Bd. 24, 1904.

29. Schlater: Histologische Untersuchungen über das Muskelgewebe. Arch. f. mikr. Anat., Bd. 69, 1907.

30. Vigier: Strukture des fibres musculaires du coeur chez les mollusques. C. R. Acad. Scienc. Paris, T. 138, 1904.

31. Vigier et Vlès: Sur l'histologie du myocard chez les mollusques. C. R. Acad. Scienc. Paris, T. 139, 1901.

32. Wiemann: The relation between the Cyto-Reticulum and the Fibrill Bundles in the Heart etc. Americ. Journ. of Anat., Vol. 8, 1907.

\section{Erklärung der Abbildungen auf Tafel XXVIII.}

Fig. 1. Alkoholpräparat. Bestsche Glykogenfärbung. - Faserbündel aus dem Froschherzen; glykogenhaltige Sarkosomen in den interkolumnären Räumen; gefärbte Querlinien in der Höhe von Z.

Fig. 2. Konservierung und Fixation wie bei 1. a, a, b = Muskelsäulchen, deren Ränder mit glykogenhaltigen Sarkosomen besetzt sind; die Mehrzahl dieser ist in der Höhe von $\mathrm{Z}$ gelegen; $\mathrm{Q}$ ungefärbt. $c=$ Muskelsäulchen von einem glykogenhaltigen Netz umsponnen.

Fig. 3. Konservierung und Fixation wie bei 1; verschiedene Abstände der roten Querlinien je nach dem Kontraktionszustand; bei b sind zu beiden Seiten des ungefärbten $\mathrm{Z}$ rote Querlinien gelegen.

Fig. 4. Konservierung nach der Bendaschen Mitochondrienmethode, Tinktion mit Eisenhämatoxylin. Zwischen den Fasern Reihen gefärbter Sarkosomen von wechselnder Grösse; stellenweise schwarze Querlinien.

Fig. 5. Konservierung und Tínktion wie bei 4 . Bei a eine durch Fäden verbundene Sarkosomenreihe, von welcher quere Fortsätze abtreten. Bei $b$ von einem schwarzen Netz umsponnenes Muskelsäulchen. Bei $c$ Verschiebung des Netzes. 
Fig. 6. Konservierung und Tinktion wie bei $4 . \quad a=$ Muskelsäulchen mit aufgelagerten Sarkosomen. $\mathrm{b}=$ Muskelsäulchen mit schwarzen in der Höhe von $\mathrm{Z}$ gelegenen Querlinien.

Fig. 7. Konservierung nach der B e nd a scher Mitochondrienmethode, Tinktion mit Krystallviolett-Anilinöl, Differenzierung mit Aceton-Nelkenöl. Bei a der mittlere Abschnitt des Fibrillensegmentes gefärbt, J ungefärbt, $\mathrm{Z}$ graublau tingiert. Bei $\mathrm{b}$ die mittleren Abschnitte der Fibrillensegmente, sowie Z und einzelne Sarkosomen gefärbt.

Fig. 8. Konservierung und Tinktion wie bei 7. An der Stelle der mittleren Abschnitte der Fibrillensegmente Stäbchen-(Myokonten), Z graublau gefärbt. $b=$ gefärbten Myokonten; an der Stelle von $Z$ gefärbte granulaartige Gebilde. $\mathrm{c}=$ isolierten Fibrillen mit gefärbten Myokonten und Z-granula. $\mathrm{d}=$ isolierten Fibrillen mit Granula: (Myosomen und Z-granula) -; die Lagen der einzelnen Granula sind nicht mit Sicherheit zu bestimmen.

Fig. 9. Konservierung and Tinktion wie bei 7 . a, b, c $=$ verschiedenen Kontraktionszuständen der Muskelsäulchen.

Fig. 10. Konservierung und Tinktion wie bei 4 ; Kern mit Karyosomen und Netzfäden. 


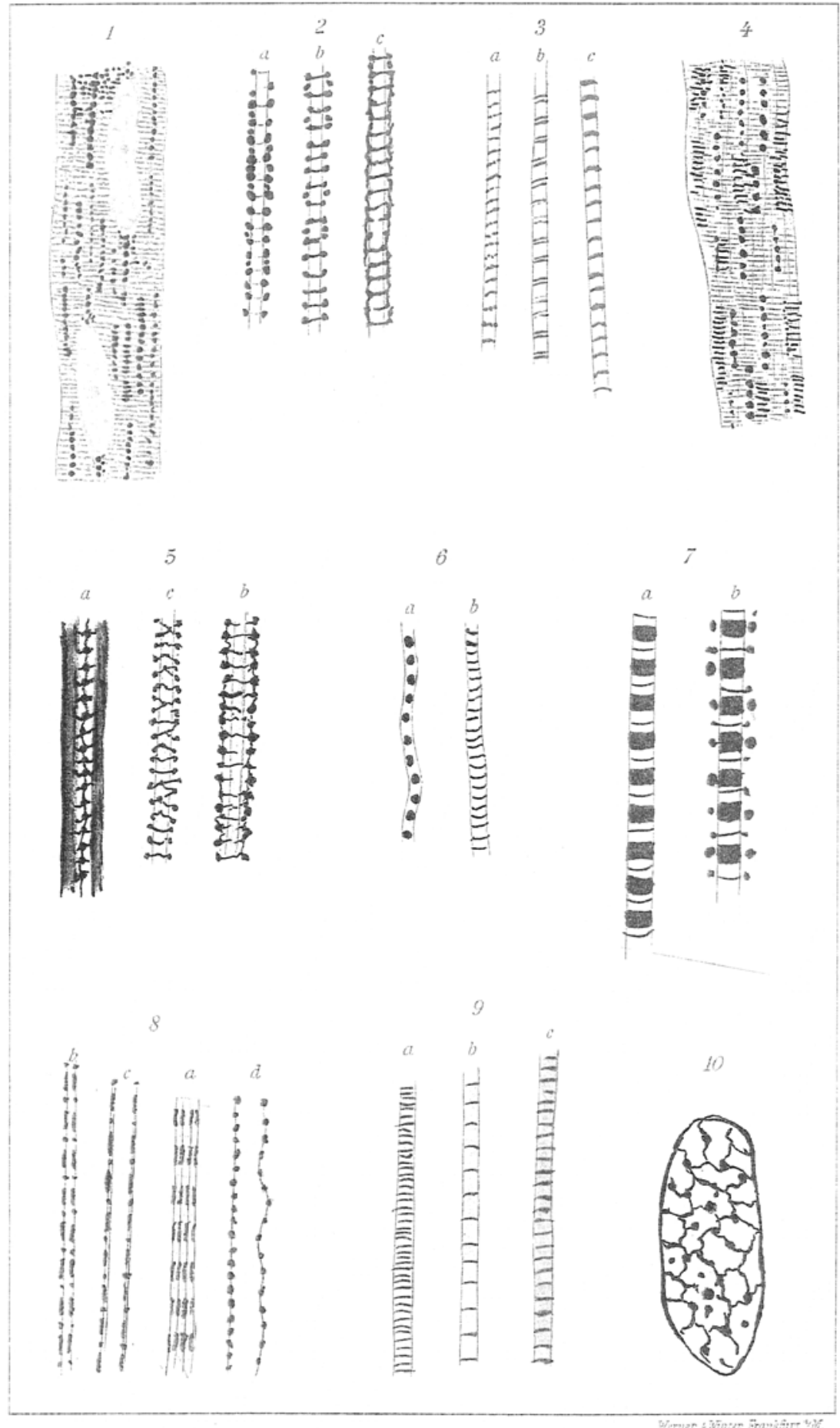

